\title{
Intrahepatic Splenosis with Severe Iron Deposition Presenting with Atypical Magnetic Resonance Images
}

\author{
Tomoki Nakajima ${ }^{1}$, Atsushi Fujiwara ${ }^{1}$, Mio Yamaguchi ${ }^{1}$, Akiko Makiyama ${ }^{1}$, Takeshi Wakae ${ }^{1}$, \\ Kinya Fujita ${ }^{1}$, Kenji Yoshikawa ${ }^{1}$, Takehiko Shiomi ${ }^{1}$, Tohru Ohishi ${ }^{1}$, Toshiaki Nakashima ${ }^{1}$ \\ and Eiichi Konishi ${ }^{2}$
}

\begin{abstract}
An intrahepatic mass was incidentally found in a 41-year-old man with a history of a traffic accident injury which resulted in removal of a ruptured spleen. Hepatic splenosis was considered in the differential diagnosis but magnetic resonance imaging showed hypointensity on T2-weighted images, atypical for normal spleen. Histologically, the mass showed sinusoidal structures and lymphoid follicular aggregates. Immunohistochemical study showed that the phenotype of the vascular lining cells was CD8-positive, CD31-positive, and CD34 negative, the pattern diagnostic for ectopic spleen. In addition, severe iron deposition was histologically demonstrated, which was considered as the cause of the hypointense T2-weighted images.
\end{abstract}

Key words: intrahepatic splenosis, ectopic spleen, iron deposition, magnetic resonance imaging

(Inter Med 47: 743-746, 2008)

(DOI: 10.2169/internalmedicine.47.0689)

\section{Introduction}

Splenosis is not a rare condition; it is estimated to occur in up to $67 \%$ of patients with splenic traumatic rupture (1). The scattering of splenic cells from the damaged spleen is the cause of the autografting of splenic tissue. Splenosis has been reported mainly in the abdominal cavity and along surgical scars, but it also can occur in the chest when the diaphragm is damaged as a consequence of trauma (2). However, there are only a limited number of reports on intrahepatic splenosis (3-16).

In contrast to accessory spleens, which are histologically and functionally identical to the normal spleen, splenosis shows some variations at the microscopic level, resulting in some variations in radiological findings (17). Splenosis occasionally can create a confusing appearance and unfortunately surgical intervention has been used to arrive at the correct diagnosis. We report a case of intrahepatic splenosis showing atypical magnetic resonance images, which was confirmed by histological evaluation of the biopsy specimen, using a combination of immunohistochemical staining of endothelial cells and iron staining.

\section{Case Report}

A 41-year-old man was admitted to our hospital, presenting with severe abdominal pain and diarrhea. He had a history of a traffic accident at age 20, which resulted in removal of a ruptured spleen. We diagnosed this condition as acute enteritis and after careful follow-up with the administration of antibiotics, the symptoms were relieved successfully. As a screening examination to determine the cause of these symptoms, we performed ultrasonographic (US) evaluation of the abdomen, which incidentally revealed a hypoechoic mass at the surface of the hepatic segment 6 . Computed tomography (CT) showed hypodensity mass protruding from the hepatic surface (Fig. 1-a). Dynamic study showed strong enhancement at the early phase (Fig. 1-b) and pooling enhancement at the late phase (Fig. 1-c). Magnetic resonance imaging (MRI) showed hypointensity on both T1- and T2-weighted images (Fig. 1-d, e). Since the

${ }^{1}$ Department of Internal Medicine, Saiseikai Kyoto Hospital, Nagaoka-kyo and ${ }^{2}$ Department of Surgical Pathology, Saiseikai Kyoto Hospital, Nagaoka-kyo

Received for publication October 23, 2007; Accepted for publication January 8, 2008

Correspondence to Dr. Tomoki Nakajima, tomnaka@silver.ocn.ne.jp 
a

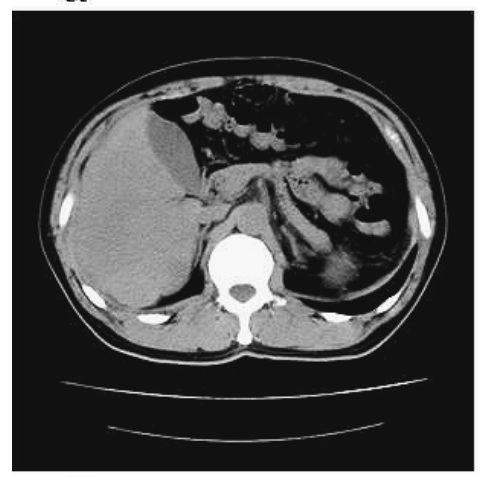

d

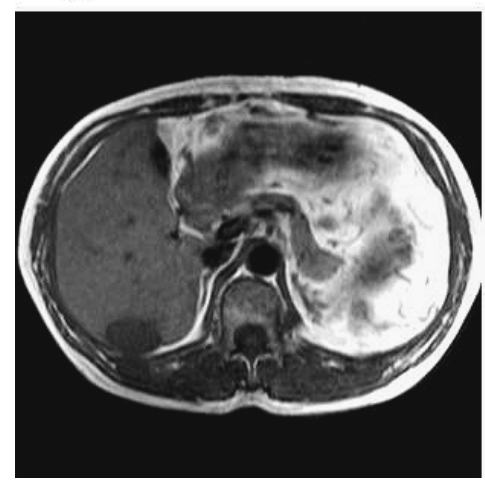

$\mathrm{b}$

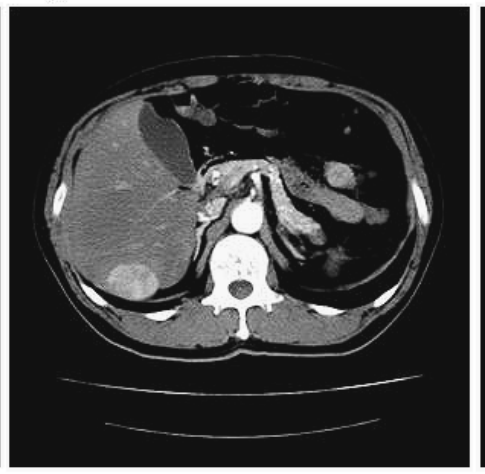

$\mathrm{e}$

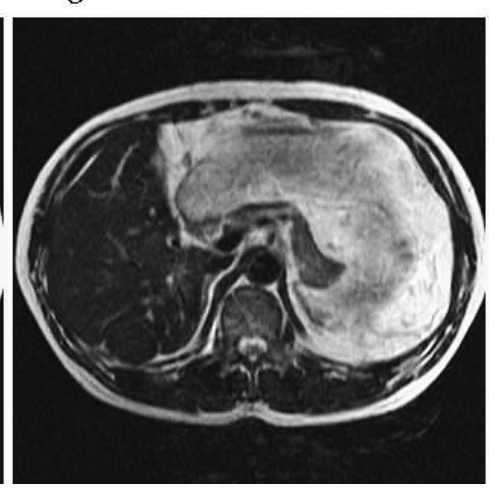

$\mathrm{C}$
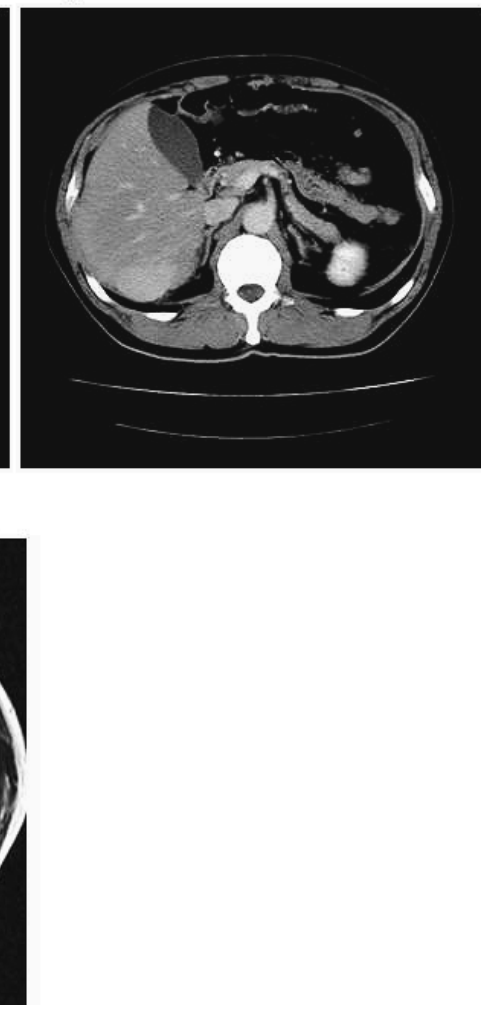

Figure 1. Computed tomography (CT) showed a hypodensity mass protruding from the surface of the hepatic segment 6 (Fig. 1-a). Dynamic study showed strong enhancement at the early phase (Fig. 1-b) and pooling enhancement at the late phase (Fig. 1-c). Magnetic resonance imaging (MRI) showed hypointensity on both T1- and T2-weighted images (Fig. 1-d and e).

liver is a possible site of autografting of splenic tissue after splenic trauma, hepatic splenosis was considered in the differential diagnosis. However, because the normal spleen generally shows hyperintensity on T2-weighted images compared with the liver, hypointensity of this mass was not compatible with the MR finding of the normal spleen. To confirm the diagnosis and elucidate the cause of this radiological finding, we performed percutaneous ultrasoundassisted liver biopsy.

The biopsy specimen was formalin-fixed and paraffinembedded. Five sections were cut serially from each paraffin block and one each was used for Hematoxylin and Eosin (HE) staining, Berlin blue staining and immunohistochemical staining for CD8, CD31 and CD34. Immunohistochemical staining was performed following the manufacturer's instruction using the DakoCytomation EnVision ${ }^{\mathrm{TM}}+$ System, Peroxidase (DakoCytomation, Carpinteria, CA). The antibodies used were anti-human CD8 monoclonal antibody (C8/144B; DakoCytomation), mouse anti-human CD31 monoclonal antibody (JC70A; DakoCytomation) and mouse anti-human CD34 monoclonal antibody (QBEnd 10; DakoCytomation). The sections were counterstained with hematoxylin.

Hematoxylin and Eosin staining showed sinusoidal structures and lymphoid follicular aggregates (Fig. 2-a). To demonstrate the origin of the sinusoidal structures, we performed immunohistochemical staining of the vascular lining cells.
The phenotype of these cells was CD8-positive, CD31positive, and CD34 negative (Fig. 2-b, c). Since this phenotypic pattern is characteristic of splenic sinusoidal lining cells (18), we histologically diagnosed this lesion as ectopic spleen. Berlin blue staining revealed marked iron deposition (Fig. 2-d). We confirmed this was the cause of hypointense T2-weighted images.

\section{Discussion}

To date, there have been only five reports on MRI of intrahepatic splenosis $(3,8,12,15,16)$. In one report, there was no description on T2-weighted images (3). In two reports, splenosis was demonstrated as hypointense on T1weighted images and hyperintense on T2-weighted images, which is similar to the normal adult spleen $(8,15,16)$. However, in the remaining one report, Lin et al described a case of splenosis which showed hypointensity on T2weighted images and speculated this to be a result of iron deposition (12).

De Vuysere et al have reported that on T2-weighted images after the injection of superparamagnetic iron oxide (SPIO), the ectopic spleen showed a 50\% loss in signal intensity but remained slightly hyperintense relative to the hypointense liver parenchyma (8). However, the lesion in the present case showed hypointensity even without SPIO injection, therefore, such SPIO-MRI finding might not be helpful 

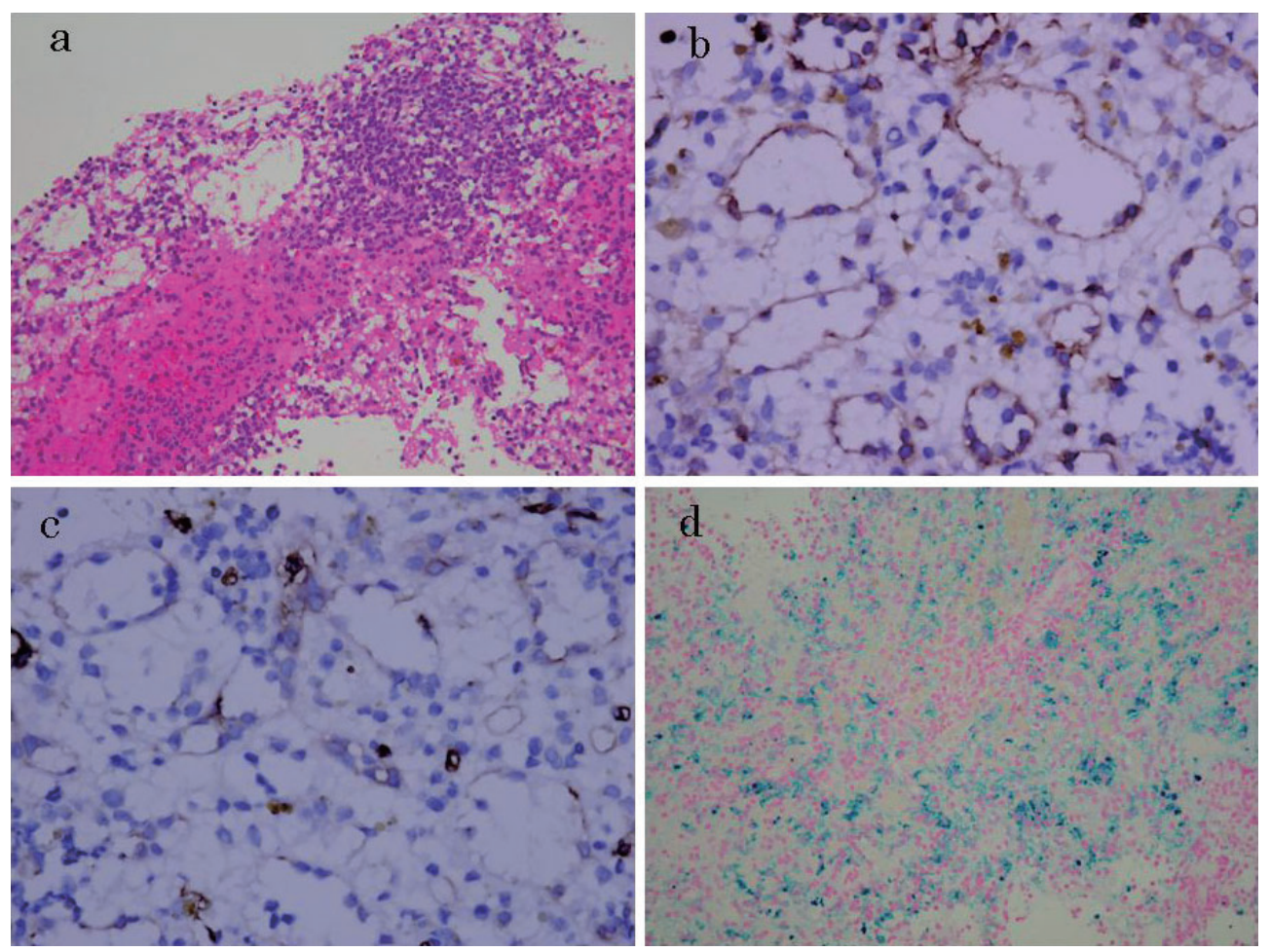

Figure 2. Hematoxylin and Eosin staining of the lesion showed sinusoidal structures and lymphoid follicular aggregates (Fig. 2-a). Immunohistochemically, the phenotype of the vascular lining cells was CD8-positive and CD34 negative (Fig. 2-b and c), which is indicative of ectopic spleen. Berlin blue staining revealed strong iron deposition (Fig. 2-d), which accounts for hypointensity on T2-weighted images (Original magnification $\times 200$ ).

for diagnosis. To clarify the origin of this lesion and the cause of this hypointensity on T2-weighted images, we performed immunohistochemical staining and iron staining, which histologically confirmed that the lesion was indeed splenic tissue accompanied with iron deposition. This is the first report to demonstrate intense iron deposition in this lesion and provide histological evidence to support the speculation by Lin et al regarding the hypointense $\mathrm{T} 2$-weighted images (12).

In contrast to accessory spleens, which are histologically and functionally identical to the normal spleen, splenosis shows some variations at the microscopic level. Carr and Turk proposed that these histological variations may depend on the local blood supply to the autotransplanted splenic tissue (17). Namely, a good blood supply to the outer portions of the tissue in the early phase after implantation may result in a well-developed splenic nodule while a poor blood supply is more likely to be associated with a poorly-developed structure. Though the exact reason is unknown, these histological variations may reflect the differences in the degree of iron deposition and consequently the variation in the signal intensity of T2-weighted images.

Since hepatocellular carcinoma and intrahepatic cholangiocarcinoma are major malignant tumors in the liver, the differential diagnosis of these tumors is clinically important. In typical cases of intrahepatic cholangiocarcinoma, the tumor is basically hypovascular. It shows ring enhancement from arterial until portal phase and prolonged enhancement at the late phase, which reflects intra-tumoral fibrosis. Because these findings are different from those of splenosis, cholangiocarcinoma is easily distinguished from hepatic splenosis. On the other hand, because of its hypervascular nature, the hepatic splenosis may mimic hepatocellular carcinoma in standard imaging techniques such as US, CT, or MRI.

When splenosis is suspected by history-taking, histology represents the gold standard for the diagnosis (14), and scintigraphy with a sensitive heat-denatured technetium-99 mlabeled red blood cells is the most specific imaging technique $(19,20)$. In the present case, to reach the final diagnosis histologically, we thought that a combination of immunohistochemical staining of the vascular lining cells and iron staining using biopsy specimen might help to avoid unnecessary surgical interventions, even in a case showing atypical MR images.

Diffusion-weighted MR imaging has recently been used to characterize abdominal organs and hepatic lesions. Ichikawa et al have shown a strong black signal in the normal spleen by providing with black-and-white reversedcontrast display of high-b-value diffusion-weighted MR images (21). Such a strong signal has been attributed to low apparent diffusion coefficient (ADC) value of the spleen. On the contrary, since the ADC values with the $b$ value of 600 $\mathrm{sec} / \mathrm{mm}^{2}$ are reported to be similar among primary liver cancer, metastatic liver cancer and the hepatic parenchyma (22), there might be no or little difference in signal intensity be- 
tween the malignant tumor in the liver and its surrounding hepatic parenchyma. Therefore, high b-value diffusionweighted MR imaging might provide useful information to differentiate between hepatic malignancies and intrahepatic splenosis.

\section{References}

1. Normand JP, Rioux M, Dumont M, Bouchard G, Letourneau L. Thoracic splenosis after blunt trauma: frequency and imaging findings. AJR 161: 739-741, 1993.

2. Fleming CR, Dickson ER, Harrison EG Jr. Splenosis: autotransplantation of splenic tissue. Am J Med 61: 414-419, 1976.

3. Yoshimitsu K, Aibe H, Nobe T, et al. Intrahepatic splenosis mimicking a liver tumor. Abdom Imaging 18: 156-158, 1993.

4. Lacerda MA, Ludwig J, Ward EM. Intrahepatic spleen presenting as a mass lesion. Am J Gastroenterol 88: 2116-2117, 1993.

5. Davidson LA, Reid IN. Intrahepatic splenic tissue. J Clin Pathol 50: 532-533, 1997.

6. D'Angelica M, Fong Y, Blumgart LH. Isolated hepatic splenosis: First reported case. HPB Surg 11: 39-42, 1998.

7. Foroudi F, Ahern V, Peduto A. Splenosis mimicking metastases from breast carcinoma. Clin Oncol (R Coll Radiol) 11: 190-192, 1999.

8. De Vuysere S, Van Steenbergen W, Aerts R, et al. Intrahepatic splenosis: imaging features. Abdom Imaging 25: 187-189, 2000.

9. Gamulin A, Oberholzer J, Rubbia-Brandt L, Mentha G. An unusual, presumably hepatic mass. Lancet 360: 2066, 2002.

10. Lee JB, Ryu KW, Song TJ, et al. Hepatic splenosis diagnosed as hepatocellular carcinoma: report of a case. Surg Today 32: 180182, 2002.

11. Kim KA, Park CM, Kim $\mathrm{CH}$, et al. An interesting hepatic mass: splenosis mimicking a hepatocellular carcinoma. Eur Radiol 13: 2713-2715, 2003.

12. Lin WC, Lee RC, Chiang JH, Wei CJ, Chu LS, Liu RS, Chang CY. MR features of abdominal splenosis. AJR Am J Roentgenol
180: 493-496, 2003.

13. Zhao M, Xu HW. Splenosis simulating an intrahepatic mass. Chin J Traumatol 7: 62-64, 2004.

14. Di Costanzo GG, Picciotto FP, Marsilia GM, Ascione A. Hepatic splenosis misinterpreted as hepatocellular carcinoma in cirrhotic patients referred for liver transplantation: report of two cases. Liver Transpl 10: 706-709, 2004.

15. Kondo M, Okazaki H, Takai K, et al. Intrahepatic splenosis in a patient with chronic hepatitis C. J Gastroenterol 39: 1013-1015, 2004.

16. Izzo L, Caputo M, Galati G. Intrahepatic accessory spleen: imaging features. Liver Int 24: 216-217, 2004.

17. Carr NJ, Turk EP. The histological features of splenosis. Histopathology 21: 549-553, 1992.

18. Yeh CJ, Chuang Wy, Kuo TT. Unusual subcutaneous splenosis occurring in a gunshot wound scar: pathology and immunohistochemical identification. Pathol Int 56: 336-339, 2006.

19. Stewart CA, Sakimura IT, Siegel ME. Scintigraphic demonstration of splenosis. Clin Nucl Med 11: 161-164, 1986.

20. Zwas ST, Samra D, Samra Y, Sibber GR. Scintigraphic assessment of ectopic splenic tissue localization and function following splenectomy for trauma. Eur J Nucl Med 12: 125-129, 1986.

21. Ichikawa T, Erturk SM, Motosugi U, et al. High-b-value diffusionweighted MRI in colorectal cancer. AJR Am J Roentgenol 187: 181-184, 2006.

22. Yoshikawa T, Kawamitsu H, Mitchell DG, et al. ADC measurement of abdominal organs and lesions using parallel imaging technique. AJR Am J Roentgenol 187: 1521-1530, 2006.

(C) 2008 The Japanese Society of Internal Medicine http://www.naika.or.jp/imindex.html 